\title{
Pumping of energy into a mesoscopic ring: Exactly solvable model
}

\author{
L. Gorelik ${ }^{1,2}$, S. Kulinich ${ }^{1,2}$, Yu. Galperin ${ }^{3}$, R. I. Shekhter ${ }^{1}$, and M. Jonson ${ }^{1}$ \\ ${ }^{1}$ Department of Applied Physics, Chalmers University of Technology and Göteborg University, \\ $S-41296$ Göteborg, Sweden \\ E-mail: gorelik@fy.chalmers.se \\ shekhter@fy.chalmers.se \\ jonson@fy.chalmers.se \\ ${ }^{2}$ B. Verkin Institute for Low Temperature Physics and Engineering, 310164 Kharkov, Ukraine \\ E-mail: kulinich@ilt.kharkov.ua \\ ${ }^{3}$ Departrment of Physics, University of Oslo, P. O. Box 1048 Blindern 0316 Oslo, Norway \\ and A. F. Ioffe Institute, 194021 St. Petersburg, Russia \\ E-mail: iouri.galperin@fys.uio.no \\ Received July 13, 1998
}

\begin{abstract}
We consider the energy stored in a one-dimensional ballistic ring with a barrier sub jected to a linearly time-dependent magnetic flux. An exact analytical solution for the quantum dynamics of electrons in the ring is found for the case when the electromotive force multiplied by the electron charge, $e \boldsymbol{\varepsilon}$, is much smaller than the interlevel spacing, $\Delta$. Electron states exponentially localized in energy space are found for irrational values of the dimensionless ratio $A \equiv \Delta / 2 e \boldsymbol{\varepsilon}$. Relaxation limits the dynamic evolution and the localization does not develop if $A$ is sufficiently close to a rational number. As a result the accumulated energy becomes a regular function of $A$ containing a set of sharp peaks at rational values with small enough denominators (fractional pumping). The shape of the peaks and the distances between them are governed by the interplay between the strength of backscattering and the relaxation rate.
\end{abstract}

PACS: 73.40.-c, 73.20.Dx

\section{Introduction}

Physical properties of mesoscopic system are strongly influenced by quantum interference of electronic states [1]. Anderson localization of electrons [2], universal fluctuations of conductance [3], as well as periodic magnetic field dependence of thermodynamic- and transport properties of multiply connected devices (e.g., metallic rings) are important examples.

Previous extensive studies in mesoscopic physics were concentrated mainly on thermodynamics, as well as on linear response of nanostructures to dc or slowly time-varying electrical and magnetic perturbations. At the same time, relatively little is known about the nonlinear response of mesoscopic systems to a time-dependent bias. In general, an electron driven by an external time-dependent force does not conserve the energy. In spite of the fact that energy is not conserved, interference processes remain cru- cially important if the phase breaking rate is much less than the rate characterizing dynamical redistribution of the electron wave function between different states in energy space.

In this paper, we consider an example of such a system, namely a single-channel mesoscopic ring subjected to a non-stationary perpendicular magnetic field, linearly dependent on time. We concentrate on the energy accumulation in such a system as a function of time. To investigate the role of interference, we take into account electron backscattering from a single potential barrier ( $\ll$ defect»), embedded in the ring. It is shown that tuning either the time derivative of the external magnetic field variation, or the transmission amplitude through the barrier (by the gate potentials) one can influence the interference pattern, and in this way change the dynamics significantly.

Disordered conducting rings have been extensively discussed in connection with energy dissipa- 
tion in mesoscopic metallic systems [4,5]. Gefen and Thouless [6,7] have suggested that randomly distributed impurities lead to the so-called dynamical localization of electrons in energy space. This phenomenon, similar to Anderson localization in real space, should exist even in ballistic rings, i.e., when the elastic mean free path is much bigger than the ring's diameter. To be more precise, the electron energy as a function of time should saturate rather than increase without bound (as is expected to happen in a perfectly ballistic ring without any impurities at all [8]). In the saturation regime the time-averaged electric current vanishes. Consequently, a slow-varying magnetic flux $\Phi(t)$ through the ring induces a circular slowly varying current only in the presence of phase breaking processes. The role of these processes was analyzed numerically in Refs. 9, 10.

Dynamical localization in energy space, as well as Anderson localization in the real space, occurs due to destructive interference of partial waves with random phases forming the electron state. However, in our case the nature of the randomness is dynamic (cf. the case of so-called kicked rotator $[11,12])$. Consequently, the interference is crucially sensitive to the rate of change of magnetic flux, scattering amplitude against the barrier, etc. As was shown in Refs. 13 and 14, for certain values of $\Phi(t)$ the energy-space propagation of the electrons can in the single-impurity case be mapped onto the real-space motion of a particle in a periodic potential. Such a Bloch-like state results in the conductance behavior qualitatively similar to the one of pure rings.

At the same time, according to numerical studies $[10,13]$ the electron appears localized in energy space at other rates of magnetic flux variation; the energy pumped into the systems saturates as expected for disordered systems. This result makes the crucial importance of the rate of change of flux clear; by tuning the time derivative of the flux (i.e., the induced electromotive force in the ring) one can crossover from one regime to the other, and in this way control the energy pumping. This is the subject of the present paper [15].

We shall show that the scenario of the crossover is as follows. Consider the conductance of the ring, $G$, defined as the ratio between the circulating current and the electromotive force $\varepsilon=-\dot{\Phi} / c$ induced in a ring of radius $r_{0}$ by a magnetic field linearly dependent on time. If scattering is strong, $G \propto \varepsilon^{-2}$. As the scattering strength decreases, a set of peaks in the $G(\varepsilon)$ dependence appears. The peaks correspond to rational values $p / q$ of the dimension- less ratio $A \equiv \Delta / 2 e \varepsilon$, where $\Delta=\hbar^{2} N_{F} / m r_{0}^{2}$. Here $N_{F}$ is the number of filled electron states while $m$ is the effective mass. The shape of the peaks as well as the distances between them are governed by an interplay between the height $V$ of the potential barrier and the relaxation rate, $v$, the maximum value of $q$ being determined by the condition $\tau^{q} / q \simeq \hbar \nu / e \boldsymbol{\varepsilon}$. Here $\tau \equiv \exp \left(-\varepsilon_{c} / \varepsilon\right)$ is the effective amplitude of Zener tunneling through the energy gaps in the electron spectrum, $\varepsilon_{c}=\pi^{2} V^{2} / 2 \Delta e$. The peak structure near a maximum can be described by the interpolation formula

$$
g=\tau^{2 q} \frac{\hbar v \Delta}{(\hbar v)^{2}+(e \varepsilon q)^{2} \varepsilon^{2}}+\eta q^{2} \frac{\hbar v \Delta}{(e \varepsilon)^{2}}, \quad \varepsilon=A-\frac{p}{q}
$$

Here $g=G / G_{0}, G_{0}=e^{2} / h$, while $\eta(\varepsilon)$ is a smooth function of $\varepsilon$. If $|\varepsilon| \leqslant \tau^{q} / q^{2}$ the function $\eta \sim 1$, beyond this region it decreases as $|\varepsilon|$ increases. As the barrier becomes more transparent, $\tau \rightarrow 1$, the inter-peak distance (determined by the maximum value of $q$ ) decreases. Finally, the peaks overlap forming the conductance $g=\Delta / \hbar v$ independent of the barrier's properties.

To understand the result conjectured above let us consider the electron energy levels in the vicinity of the Fermi level, $E_{F}$, where the energy dispersion can be considered as linear. In a ballistic ring, one has then two sets of adiabatic energies $E_{l}(\Phi)$ corresponding to clock- and counterclockwise motion (Fig. 1). The scattering from the barrier opens gaps for the flux values $E_{l}=E_{F}+l \Delta / 2, l=0, \pm 1, \pm 2, \ldots$ the energy levels for clockwise and counterclockwise motion coincide. Consequently, the energy pumping into the system by a slowly varying magnetic flux can be mapped onto the one-dimensional motion of a quantum particle in the field of periodically placed scatterers (cf. Refs. 6, 7, 13). Landau-Zener tunneling (with the amplitude $\tau$ introduced above) through the gaps corresponds to forward scattering while reflection from the gaps is

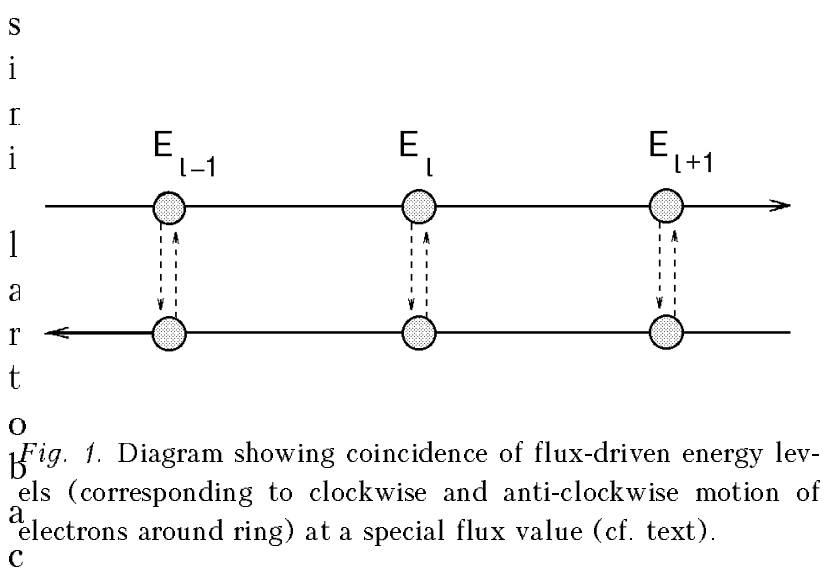


kscattering. The important difference from the usual impurity problem is that there is no translational invariance at an arbitrary value of the driving force $\varepsilon$. This invariance is only present for rational values $p / q$ of the dimensionless ratio $A$ [13]. In this case we arrive at a superlattice containing $q$ «impurities» per unit cell. As a result, the motion along $E$-axis is described by $q$ allowed bands, the «velocity» being. $v_{E} \equiv \dot{E} \sim \Delta \tau^{q} / t_{0} \quad$ (here $t_{0} \equiv h / e \varepsilon=\Phi_{0} / \dot{\Phi}$ is twice the time interval between two sequential Landau-Zener scattering events). Since the upper bound of the Brillouin zone is $4 \pi \hbar / q \Delta$, the corresponding bandwidth for the motion along $E$-axis is $W \simeq v_{E} 4 \pi \hbar / q \Delta \simeq$ $\simeq 4 \pi \hbar \tau q / t_{0} q$. At rational values $p / q$ of the quantity $A$ the electron experiences $2 p$ rotations around the ring while the enclosed magnetic flux changes by $q$ quanta. As a result the «motion» of the system along $E$-axis can be mapped onto the motion of a quantum particle in a one-dimensional periodic potential, the corresponding eigenstates being extended. If $p / q$ is irrational the equivalent potential is quasi-periodic. It turns out that in such a case the corresponding states are then localized (see below) in spite of the fact that there is no real disorder in the system. The localization length in energy space, $R_{\text {loc }}$, can be estimated for $A=p / q+\varepsilon,|\varepsilon|<<1 / q$ as follows. At finite $\varepsilon$ the phase mismatch with respect to the case of rational $A=p / q$ can be ascribed to a quasiclassical potential $U(E)=\varepsilon \alpha E$ with $\alpha=8 \pi \hbar / \Delta t_{0}$. This potential gives rise to band bending which creates semiclassical turning points for the modes propagating along the $E$-axis. The localization length can be estimated as half the distance between the turning points produced by the upper and lower band edges, $R_{\text {loc }} \simeq W / 2 \alpha|\varepsilon|=$ $=\Delta \tau^{q} / 4 q|\varepsilon|$. Consequently, the localization time is $t_{\mathrm{loc}} \sim 4 R_{\mathrm{loc}} / v_{E} \sim t_{0} / q|\varepsilon|$.

The manifestation of localization in the energy pumping depends on the product $v t_{\mathrm{loc}}$. At $v t_{\text {loc }}>>1$ localization has no chance to develop and the band picture of energy pumping is relevant. The conductance is estimated as (cf. with Ref. 7) $G=P / \varepsilon^{2}$, where $P$ is the average energy accumulation rate. The quantity $P$, in its turn, is determined as $\mathrm{v}(\delta E) N_{\text {eff }}$. Here $\delta E \sim v_{E} / \mathrm{v}$ is the energy accumulated by a single state, while $N_{\text {eff }}(\delta E) \sim \delta E / \Delta$ is the number of involved states. It follows that $g \sim \tau^{2 q}(\Delta / \hbar v)$. If $v t_{\text {loc }}<1$, on the other hand, $G$ is determined by hops between intraband localized states. In this case, $\delta E \sim 2 R_{\text {loc }}$, and we obtain $g \sim \hbar \vee \Delta \tau^{2 q} /(e \varepsilon q \varepsilon)^{2}$. These estimates are consistent with the first term in Eq. (1).
The paper is organized as follows. In the following section the theoretical model is described and basic expressions for the electrical current are obtained. These expressions are analyzed and discussed in Sec. 3, the details of calculations being outlined in Appendices.

\section{Theory}

\section{A. The model}

The following model is employed. The electron system is described by the Hamiltonian

$$
\mathcal{H}(t, \varphi)=-\Delta \sigma_{z}\left(i \frac{\partial}{\partial \varphi}+\frac{t}{t_{0}}\right)+\mathcal{H}_{\mathrm{imp}}(\varphi)
$$

Here $\sigma_{i}$ are Pauli matrices. We are interested in the current, averaged over the time $t_{0}$,

$$
I(t)=\frac{1}{t_{0}} \int_{t_{-}}^{t_{+}} d t^{\prime} \operatorname{Tr} \hat{\rho} \hat{j}
$$

where $t_{ \pm}=t \pm t_{0} / 2$

$$
\hat{j} \equiv(i e / \hbar)[\mathcal{H}, \hat{\varphi}]
$$

is the current operator. The single-electron density matrix, $\rho$, is calculated from the equation

$$
\frac{\partial \hat{\rho}}{\partial t}=\frac{i}{\hbar}[\hat{\rho}, \mathcal{H}]-v\left(\hat{\rho}-f_{0}[\mathcal{H}(t)]\right),
$$

where $f_{0}$ is the Fermi function.

\section{B. The average current}

The formal solution of Eq. (4) can be expressed in terms of the evolution operator $\hat{u}\left(t, t^{\prime}\right)$, which satisfies the equation

$$
i \hbar \frac{d \hat{u}\left(t, t^{\prime}\right)}{d t}=\mathcal{H} \hat{u}\left(t, t^{\prime}\right)
$$

with the initial condition $\hat{u}(t, t)=1$. It has the form

$$
\hat{\rho}(t)=v \int_{-\infty}^{t} d t^{\prime} \mathrm{e}^{v\left(t^{\prime}-t\right)} \hat{u}\left(t, t^{\prime}\right) f_{0}\left[\mathcal{H}\left(t^{\prime}\right)\right] \hat{u}^{+}\left(t, t^{\prime}\right) .
$$

The average current can be expressed through the Heisenberg operators for the current, $\hat{j}(t)$, and the velocity in energy space, $\hat{v}(t)$,

$$
\begin{gathered}
\hat{j}(t)=\hat{u}^{+}(t, 0) \hat{j} \hat{u}(t, 0), \\
\hat{v}(t)=\hat{u}^{+}(t, 0) \dot{f}_{0}[\mathcal{H}(t)] \hat{u}(t, 0)
\end{gathered}
$$


as

$$
I(t)=\frac{1}{t_{0}} \int_{t_{-}}^{t_{+}} d t^{\prime} \int_{-\infty}^{t^{\prime}} d t^{\prime \prime} \mathrm{e}^{-v\left(t^{\prime}-t^{\prime \prime}\right)} \operatorname{Tr}\left[\hat{j}\left(t^{\prime}\right) \hat{v}\left(t^{\prime \prime}\right)\right] .
$$

Using the symmetry property of the Hamiltonian (2)

$$
\mathrm{e}^{i \hat{\varphi}} \hat{\mathcal{H}}(t, \varphi) \mathrm{e}^{-i \hat{\varphi}}=\hat{\mathcal{H}}\left(t+t_{0}, \varphi\right)
$$

one obtains the symmetry properties of the operators $\hat{u}, \hat{\rho}$ and $\hat{v}$ :

$$
\begin{gathered}
\hat{u}\left(t+t_{0}, t^{\prime}+t_{0}\right)=\mathrm{e}^{\hat{i \varphi}} \hat{u}\left(t, t^{\prime}\right) \mathrm{e}^{-i \hat{\varphi}}, \\
\hat{\rho}\left(t+t_{0}\right)=\mathrm{e}^{i \hat{\varphi}} \hat{\rho}(t) \mathrm{e}^{-i \hat{\varphi}}, \\
\hat{v}\left(t+t_{0}\right)=\hat{T}^{+} \hat{v}(t) \hat{T},
\end{gathered}
$$

where operator $\hat{T}$ is

$$
\hat{T}=\mathrm{e}^{-i \hat{\varphi}} \hat{u}\left(t_{0}, 0\right) .
$$

Using (7) and (8) it is easy to prove that the average current (3) is time-independent, $I(t)=$ const $\equiv I$. Having in mind the symmetry properties (7) and (8) it is convenient to divide the integration interval $\left(-\infty, t^{\prime}\right)$ in (6) into the intervals $-t_{0} / 2-m t_{0}<t^{\prime \prime}<t_{0} / 2-m t_{0}, \quad m=0,1, \ldots \quad$ In this way one can express the average current $I$ as

$$
I=\sum_{m=0}^{\infty} \mathrm{e}^{-2 \tilde{v} m} \operatorname{Tr}\left(\hat{T}^{-m} \hat{J} \hat{T}^{m} \hat{\mathcal{F}}\right)-\operatorname{Tr} \hat{J}_{1},
$$

where $\tilde{v} \equiv v t_{0} / 2$, while

$$
\begin{gathered}
\hat{J}=\frac{1}{t_{0}} \int_{-t_{0} / 2}^{t_{0} / 2} d t \mathrm{e}^{-v t} \hat{j}(t), \\
\hat{\mathcal{F}}=\int_{-t_{0} / 2} d t \mathrm{e}^{v t} \hat{v}(t), \\
\hat{J}_{1}=\frac{1}{t_{0}} \int_{-t_{0} / 2} d t \int_{t}^{t_{0} / 2} d t^{\prime} \mathrm{e}^{-v\left(t-t^{\prime}\right)} \hat{j}(t) \hat{v}\left(t^{\prime}\right) .
\end{gathered}
$$

Thus the average current is expressed in terms of the operators $\hat{J}, \hat{\mathcal{F}}, \hat{J}_{1}$ defined along the time interval $-t_{0} / 2, t_{0} / 2$ which are dependent on the dynamics between the successive Landau-Zener tunneling events, and by the operator $\hat{T}$ which describes the long-time dynamics. Below we shall show that long-time behavior of the system is actually determined by the eigenstates $|\beta\rangle$ and eigenvalues $\exp (i \beta)$ of the unitary operator $\hat{T}$.

\section{Analysis of the operator $\hat{T}$}

As was shown above, one has to analyze the unitary operator $\hat{T}$ in order to understand the longtime dynamics. Its eigenstates and eigenvalues are determined by the equation

$$
\hat{T}|\beta\rangle=\exp (i \beta)|\beta\rangle \text {. }
$$

In the following, we restrict ourselves to the case of weak scattering, i.e., we assume that the relevant matrix element $V$ (which corresponds to a momentum transfer $\sim 2 p_{F}$ ) is much smaller than the interlevel spacing, $\Delta$. For $|V|<<$ the impurity potential is important only near the crossings of «clockwise» and «counterclockwise» adiabatic terms, that takes place at the times $t_{m}=m t_{0} / 2$. As a result of scattering, gaps are created in the adiabatic spectrum at $t=t_{m}$. Beyond the adiabatic approximation, these gaps can be penetrated by Landau-Zener tunneling.

Consequently, one can discriminate between rather large time intervals of ballistic evolution (with the duration $\sim t_{0} / 2$ ) and small intervals of Landau-Zener tunneling. The typical duration of the later intervals is $\leqslant \sqrt{t_{0} \hbar / \Delta}$ (cf. with Ref. 16). Thus at $\Delta t_{0} / \hbar>>1$ the Landau-Zener tunneling is indeed essentially confined within narrow intervals and therefore can be described in terms of the scattering matrix

$$
\hat{S}=\exp \left(i \theta_{0}\right)\left[\sqrt{1-\tau^{2}} \exp \left(i \theta_{1} \sigma_{z}\right)+i \tau \sigma_{x}\right] .
$$

The physical meaning of $\tau$ is the probability amplitude for Landau-Zener «forward scattering», i.e., to the transition into the state with the same angular moment while traversing the interval of non-adiabatic motion. It turns out that the quantities of interest here are independent of the phases $\theta_{0}$ and $\theta_{1}$. For simplicity we put $\theta_{0}=\theta_{1}=0$.

Having in mind the periodicity of $\varphi$ we introduce the vector basis

$$
|n, \pm\rangle \equiv \frac{\mathrm{e}^{ \pm i\left(N_{F}+n\right) \varphi}}{\sqrt{2 \pi}} \mathbf{s}_{ \pm}, \quad \mathbf{s}_{+}=\left(\begin{array}{l}
1 \\
0
\end{array}\right), \quad \mathbf{s}_{-}=\left(\begin{array}{l}
0 \\
1
\end{array}\right) .
$$

In this representation, the operator $\hat{T}$ (9) can be treated as an operator acting on direct product $n$ and pseudo-spin $(s \equiv \pm)$ spaces, 


$$
\begin{aligned}
& \hat{T}=\sqrt{1-\tau^{2}} \hat{S} \mathrm{e}^{i \pi a \sigma_{z} \otimes \mathrm{e}^{4 i \pi a \hat{n}}}+ \\
& +i \tau \sum_{ \pm} \hat{S} \sigma_{ \pm} \otimes \mathrm{e}^{2 i \pi a \hat{n}} \hat{R}_{ \pm} \mathrm{e}^{2 i \pi a \hat{n}}
\end{aligned}
$$

The operators $\hat{R}_{ \pm}$and $\hat{n}$ are defined as

$$
\hat{R}_{ \pm}|n, s\rangle=|n \mp 1, s\rangle, \quad \hat{n}|n, s\rangle=n|n, s\rangle,
$$

$a$ is the fractional part of the quantity $A=\Delta / 2 e \boldsymbol{\varepsilon}$ introduced above, while $\sigma_{ \pm}=\left(\sigma_{x} \pm i \sigma_{y}\right) / 2$.

The most interesting situation is the case of weak relaxation, $v t_{0}<<1$. This inequality means that the relaxation time is much longer than the interval between successive Landau-Zener transitions. It can be shown by a direct calculation that if this inequality is met then the operators $\hat{J}$ and $\hat{\mathcal{F}}$ acquire the form [17]

$$
\begin{gathered}
\hat{J}=I_{0} \hat{V} \otimes \delta_{n, n^{\prime}}, \\
\hat{\mathcal{F}}=\hat{V} \otimes \delta_{0, n} \delta_{0, n^{\prime}},
\end{gathered}
$$

where $I_{0}=|e| \Delta / \hbar$ is the amplitude of the persistent current while

$$
\hat{V}=\frac{1}{2}\left(\sigma_{z}-\hat{S} \sigma_{z} \hat{S}^{+}\right)=\tau^{2} \sigma_{z}-\tau \sqrt{1-\tau^{2}} \sigma_{y} .
$$

Under the same conditions $\operatorname{Tr} \hat{J}_{1}=I_{0} \tau^{2}$.

The unitary operator $\hat{T}$ (as it can be shown by direct calculation) possesses the properties:

$$
\hat{R}_{-} \hat{T} \hat{R}_{+}=\mathrm{e}^{-4 i \pi a} \hat{T}, \quad \sigma_{y} \hat{T}^{*}(-\hat{n}) \sigma_{y}=\hat{T}(\hat{n}) .
$$

These properties result in the following relations between the eigenstates of the operator $\hat{T}$

$$
\begin{gathered}
\boldsymbol{\Psi}_{\beta}(n+m)=\boldsymbol{\Psi}_{\beta-4 \pi a m}(n), \quad m=0, \pm 1, \ldots, \\
\sigma_{y} \boldsymbol{\Psi}_{\beta}^{*}(-n)=\boldsymbol{\psi}_{-\beta}(n) .
\end{gathered}
$$

At irrational values of $a$ these relations allow one to generate a complete set of eigenstates provided $\boldsymbol{\Psi}_{\beta_{0}}$ is known (see Appendix A). Hence, in this case the spectrum of $\hat{T}$ can be expressed in the form $\exp \left(i \beta_{r}^{ \pm}\right)$, where

$$
\beta_{r}^{ \pm}= \pm \beta_{0}(a)-4 \pi a r, \quad r=0, \pm 1, \pm 2, \ldots
$$

At the same time it is evident that at rational values $a=p / q$ the operator $\hat{T}$, according to (14), possesses the translational symmetry, $\hat{R}_{ \pm}^{q} \hat{T} \hat{R}_{ \pm}^{-q}=\hat{T}$. Consequently, the eigenstates of the operator $\hat{T}$ have the Bloch form while the spectrum of $\hat{T}$ can be represented by a band structure. In this case the relations
(16) also generate a complete set of eigenstates provided structure of one band is known.

Using (10), (12) one can express the average current in terms of eigenvalues and eigenfunctions of the operator $\hat{T}$

$$
\frac{I}{I_{0}}=-\tau^{2}+\sum_{\beta, \beta^{\prime}, n} \frac{\left(\psi_{\beta}(n), \hat{V} \psi_{\beta^{\prime}}(n)\right)\left(\Psi_{\beta^{\prime}}(0), \hat{V} \psi_{\beta}(0)\right)}{1-\exp \left[-2 \tilde{v}+i\left(\beta^{\prime}-\beta\right)\right]},
$$

where we denote $(\mathbf{a}, \mathbf{b})$ the scalar product in pseudo spin space.

Using the equality $\hat{V}^{2}=\tau^{2}$ originating from the definition (15) and the properties (16) of the eigenfunctions $\boldsymbol{\psi}_{\beta}(n)$ we can express the constant $\tau^{2}$ in the form

$$
2 \tau^{2}=\sum_{\beta, \beta^{\prime}}\left|\left(\Psi_{\beta}(n), \hat{V} \Psi_{\beta^{\prime}}\left(n^{\prime}\right)\right)\right|^{2},
$$

(for any $n, n^{\prime}$ ). In the limit $\tilde{v}<<1$ we get the following expression for the average current

$$
\frac{I}{I_{0}}=\sum_{\beta}\left|\Omega_{\beta}\right|^{2} \frac{\tilde{\mathrm{v}}}{\tilde{\mathrm{v}}^{2}+\sin ^{2} \Phi_{\beta}},
$$

where

$$
\Omega_{\beta}=\sum_{n}\left(\Psi_{\beta_{0}}(n), \hat{V} \Psi_{\beta}(n)\right), \quad \Phi_{\beta}=\frac{1}{2}\left(\beta-\beta_{0}\right)
$$

and $\boldsymbol{\psi}_{\beta_{0}}(n)$ is any solution of the Eq. (12) (as follows from (16), the expression (18) does not depend on the choice of $\beta_{0}$ ).

To find the eigenstates and eigenvalues of the operator $\hat{T}$ explicitly it is convenient to introduce the operators

$$
\begin{aligned}
& \hat{U}_{ \pm}=\hat{I}+\frac{2 \mathrm{e}^{i \pi a / 2}}{\tau} \sigma_{ \pm} \otimes \sin \left(2 \pi a n-\frac{\beta \pm \pi a}{2}\right), \\
& \hat{L}=\sigma_{-} \sigma_{+} \otimes \mathrm{e}^{i \pi a n}+\hat{S} \sigma_{+} \sigma_{-} \otimes \mathrm{e}^{-i \pi a n+i \beta / 2}
\end{aligned}
$$

As follows from the definition (19)

$$
\hat{U}_{ \pm}^{-1}=\hat{I}-\frac{2 \mathrm{e}^{i \pi a / 2}}{\tau} \sigma_{ \pm} \otimes \sin \left(2 \pi a n-\frac{\beta \pm \pi a}{2}\right),
$$

$\hat{L}^{-1}=\frac{1}{\sqrt{1-\tau^{2}}}\left(\sigma_{-} \sigma_{+} \hat{S}^{+} \otimes \mathrm{e}^{-i \pi a n}+\sigma_{+} \sigma_{-} \otimes \mathrm{e}^{i \pi a n-i \beta / 2}\right)$.

By direct calculations one can show that the operator $\hat{T}-\mathrm{e}^{i \beta}$ can be rewritten in the form

$$
\hat{T}-\mathrm{e}^{i \beta}=\hat{Q}\left[\hat{R}_{+} \hat{U}_{+}+\hat{U}_{-}\right] \hat{L}^{-1},
$$


where the operator $\hat{Q}$ is

$$
\begin{aligned}
& \hat{Q}=\left(i \tau \hat{S} \sigma_{-} \otimes \mathrm{e}^{2 i \pi a n} \hat{R}_{-} \mathrm{e}^{2 i \pi a n}\right) \hat{L} \hat{U}_{-}^{-1}+ \\
& +\left(i \tau \hat{S} \sigma_{+} \otimes \mathrm{e}^{2 i \pi a n} \hat{R}_{+} \mathrm{e}^{2 i \pi a n}\right) \hat{L} \hat{U}_{+}^{-1} \hat{R}_{-} .
\end{aligned}
$$

From the definition (20) and Eq. (12) one obtains the equation for the auxiliary function $\mathbf{d}_{\beta}(n)=$ $=\hat{L}^{-1} \boldsymbol{\Psi}_{\beta}(n)$,

$$
\left[\hat{R}_{+} \hat{U}_{+}+\hat{U}_{-}\right] \mathbf{d}_{\beta}(n)=0,
$$

which is equivalent to the set of equations for the components $d_{ \pm, \beta}(n) \equiv\left(\mathbf{s}_{ \pm}, \mathbf{d}_{\beta}(n)\right)$. The set reads as

$$
\begin{gathered}
d_{ \pm, \beta}(n)+d_{ \pm, \beta}(n \mp 1)+ \\
+\frac{2 \mathrm{e}^{i \pi a / 2}}{\tau} \sin \left[2 \pi a n-\frac{\beta \pm \pi a}{2}\right] d_{\mp, \beta}(n)=0 .
\end{gathered}
$$

Introducing the function

$$
B(m)= \begin{cases}d_{+, \beta}\left(\frac{m}{2}\right) & \text { if } m \text { is even } \\ d_{-, \beta}\left(\frac{m+1}{2}\right) & \text { if } m \text { is odd }\end{cases}
$$

one can reduce the set of difference equations (21) to a single equation for $B(m)$

$$
\begin{gathered}
B(m+1)+B(m-1)+ \\
+\frac{2 \mathrm{e}^{i \pi a / 2}}{\tau} \sin \left(\pi a m+\frac{\beta-\pi a}{2}\right) B(m)=0 .
\end{gathered}
$$

Thus, the vector equation (12) is reduced to the scalar equation (23) for $B(m)$. Its solution allows one to determine both the eigenstates and eigenvalues of $T$ (see Appendix B).

The results are different for the cases of rational and irrational $a$.

1. Case of irrational a-values. According to our analysis (see Appendix B)

$$
\beta=\beta_{r}^{ \pm}= \pm \pi a-4 \pi a r, \quad r=0, \pm 1, \pm 2, \ldots
$$

At $\beta=\pi a$ the eigenstate has the form

$$
\Psi_{\pi a}=\frac{\mathrm{e}^{\mp i \pi a n(2 n \pm 1)}}{2 \pi} \int_{0}^{2 \pi} d k \mathrm{e}^{-i k(2 n-1)}\left(\begin{array}{l}
\mathrm{e}^{-i k+i \chi(k)} \\
\mathrm{e}^{-i \chi(k-\pi a)}
\end{array}\right),
$$

where

$$
\chi(k)=-\sum_{l=1}^{\infty} \frac{\tau^{l}}{l} \frac{\cos l(k-\pi / 2)}{\sin \pi a l} .
$$

The infinite series (26) converges for almost all irrational values of $a$, and $\chi(k)$ is an analytic function (see Appendix B). Consequently, all eigenfunctions are exponentially localized, the localization length $R_{\text {loc }}$ in energy space being

$$
\begin{gathered}
R_{\text {loc }}^{2}=\Delta^{2}\left[\left\langle\beta\left|\hat{n}^{2}\right| \beta\right\rangle-\langle\beta|\hat{n}| \beta\rangle^{2}\right]= \\
=\left(\frac{\Delta}{2}\right)^{2}\left[\sum_{l=1}^{\infty} \frac{\tau^{2 l}}{\sin ^{2} \pi a l}+O(1)\right] .
\end{gathered}
$$

One can see that in the vicinity of rational values $p / q$ of a the localization length $R_{\text {loc }}$ diverges as $\Delta \tau q /(2 \pi q|a-p / q|)$ in agreement with the qualitative estimates given above.

2. Rational values of $a(=p / q)$. Since the problem is translationally invariant in $n$-space the eigenstates can be labeled by a quasi momentum $\mathcal{K}$ $(0 \leq \mathcal{K}<2 \pi / q)$. The spectrum is now given by [18] $\beta \equiv \beta_{r}^{ \pm}(\mathcal{K})$,

$$
\begin{gathered}
\beta_{r}^{ \pm}(\mathcal{K})=2 \pi a\left(r+\frac{1}{2}\right) \pm \\
\pm \frac{2}{q} \arcsin \left\{\tau^{q} \sin \frac{q}{2}(\mathcal{K}-2 \pi a r+\pi)\right\},
\end{gathered}
$$

where $r=0,1, \ldots, q-1$.

An expression for the Bloch function $\psi_{\beta}(n)$ for the case of rational $a$-values is given in Appendix B.

\section{Final results}

In the strong localization limit $(\tilde{v} \rightarrow 0)$ one obtains the following expression from Eq. (18) for the current (and dimensionless conductance) (see Appendix C)

$$
I=I_{0} \tilde{v}\left(\frac{2}{\Delta} R_{\mathrm{loc}}\right)^{2}, \quad g=\pi h v \frac{\Delta}{(e \varepsilon)^{2}}\left(\frac{2}{\Delta} R_{\mathrm{loc}}\right)^{2} .
$$

Consequently, in the «irrational» case the average current tends to zero, when $\tilde{v} \rightarrow 0$.

In the «rational» case the current can be expressed as

$$
\frac{I}{I_{0}}=\frac{1-\sqrt{1-\tau^{2 q}}}{\tilde{v}}+\sum_{ \pm, r=0}^{q-1} d \mathcal{K} \mid \Omega_{r}^{ \pm} \mathcal{K}^{2} \frac{\tilde{\mathrm{v}}}{\tilde{\mathrm{v}}^{2}+\sin ^{2} \Phi_{r}^{ \pm}}
$$




$$
\Phi_{r}^{ \pm}=\frac{\beta_{0}^{+}(\mathcal{K})-\beta_{r}^{ \pm}(\mathcal{K})}{2}, \quad \Omega_{r \mathcal{K}}^{ \pm}=\sum_{n=0}^{q-1}\left(\phi_{\beta_{0}}, \hat{V} \phi_{\beta_{r}^{ \pm}}\right.
$$

with the help of (16) and (27). Here $\phi_{\beta_{r}^{ \pm}}(n)$ is the Bloch amplitude corresponding to the eigenstate $\boldsymbol{\Psi}_{r}^{ \pm} \mathcal{K}^{(n)}$. The first term in Eq. (29) determines the contribution from the intraband transitions to the average current. Formally, it tends to infinity when $\tilde{v} \rightarrow 0$. Consequently, the intraband transitions determine the singular behavior of the current in the rational case. These conclusions are in agree-

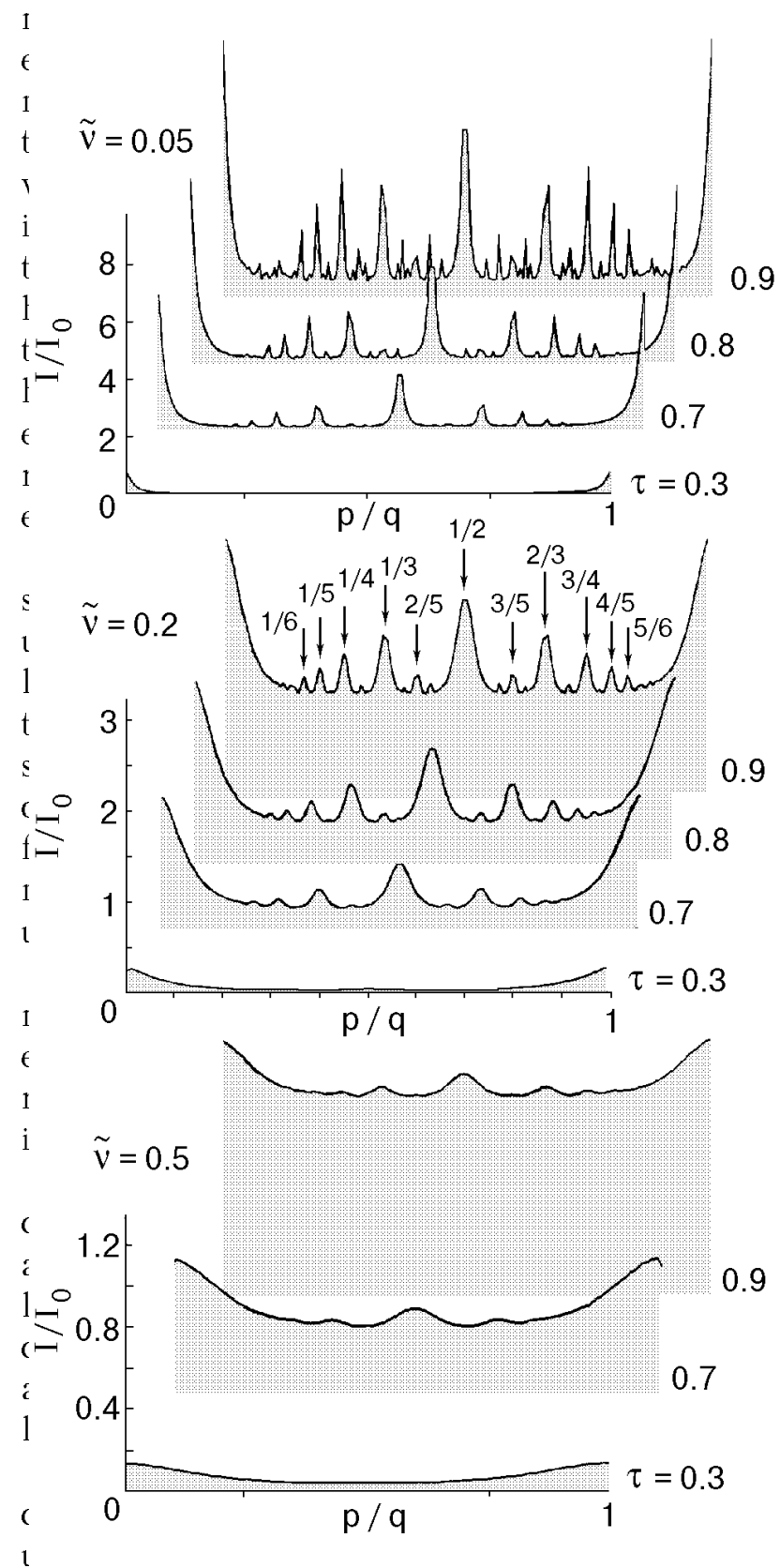

Fig. 2. The normalized current $I / I_{0}$ as a function of $a=p / q$ for different Landau-Zener tunneling amplitudes $\tau, I_{0}=k \mid \Delta / \hbar$. The values $\tilde{v}=0.05$ (upper panel), 0.2 (middle panel), 0.5 (lower panel) for the dimensionless relaxation rate was used. Arrows indicate the positions of maxima. tions in Ref. 13.

An exact expression for the eigenfunction $\phi_{\beta_{r}^{ \pm}(n)}$ shows the limiting transition to the expression (25) as $q, p \rightarrow \infty, p / q=$ const. The current calculated according to Eq. (29) also remains continuous. Thus, Eq. (29) (with large enough $p$ and $q$ ) can be used as a good approximation for irrational $a$-values. The results of such a calculation are shown in Fig. 2.

\section{Discussion and conclusion}

The following two assumptions have been implicitly made in our consideration: (i) the electron dynamics are governed by a linear dispersion law; (ii) the energy gaps as well as the scattering matrices $S$ are the same for all the energy levels involved.

Assumption (i) is valid if the number of involved states (limited by the relaxation rate) is much smaller than $N_{F}$. This is the case if $(\hbar v / \Delta)^{2}(\Delta / e \varepsilon) N_{F}>>1$. The first factor in this product is small while the two others are large. However, it can be concluded that the criterion can still be met under realistic experimental conditions.

Assumption (ii) is valid if the Fourier component of the impurity (barrier) potential, $V_{n} \equiv \int V(\varphi) \mathrm{e}^{2 i n \varphi} d \varphi$, is essentially $n$-independent for the relevant interval of $n, \delta n \leq 1 / v t_{0}$. This is the case if the scattering potential is confined to a region of width $\delta \varphi<<2 \pi v t_{0}$. Note that the inequality $v t_{0}<1$ is essential for maintaining a noticeable energy pumping.

If there are two point scatterers in the system the distance between them may be treated as the width of one barrier potential. So in the case of well separated impurities $(\delta \varphi \sim 1)$ the interference from different point scatterers will generate a quasiperiodic dependence of the gaps $V_{n} \propto \cos (n \delta \varphi)$. This circumstance will generate an additional quasiperiodic disorder in scattering (nondiagonal disorder). The problem whether this type of disorder is enough for localizing electrons in energy space at any value of the flux rate needs further considerations. Nowever, for an increasing number of scatterers the randomness in the energy gap distribution will increase tending to make the distribution truly random. This would strongly affect the «fractional» regime of energy pumping and presumably suppress the absorption peaks in Fig. 2. This crossover from the picture considered in this paper to a case of more than one scatterer is a way to introduce disorder into the problem [6].

Another approximation is that we have allowed for relaxation in the simplest possible way by using a single relaxation time in the Eq. (4) for the 
density matrix. This assumption is adequate if the relaxation is caused by a transfer of the electrons in real space between the ring and a surrounding reservoir. If the electron energy spectrum in the reservoir is continuous, then the lifetime of an electron state in the ring with respect to this mechanism is almost independent of its quantum numbers. The mechanism discussed above allows us to describe electron states in the ring as pure quantum states, the relaxation rate being the decay through escape to the reservoir. The exact results obtained above are relevant for the case when such an «escape» mechanism dominates. Internal inelastic relaxation processes in the ring can in principle lead to a significant difference between phase- and energy relaxation rates. Such a situation requires a separate treatment. However, in the most interesting case of efficient Landau-Zener tunneling, the intrinsic inelastic processes must involve large momentum transfer and therefore they are strongly suppressed [8].

In conclusion, the quantum electron dynamics problem in a single-channel ballistic ring with a barrier, subjected to a linearly time-dependent magnetic flux has been solved exactly. Exponential localization in energy space has been proven. Finally, we have shown that the dc-current exhibits a set of peaks with fractional structure when plotted as a function of the induced electromotive force. This structure is strongly sensitive to the barrier height, as well as to the relaxation rate.

This work was supported by the Swedish Royal Academy of Sciences (KVA), The Swedish Research Council for Engineering Sciences (TFR) and by the Research Council of Norway. We also acknowledge partial financial support from INTAS grant No 94-3862.

\section{Appendix A}

To prove that the functions $\left\{\psi_{\beta}(n)\right\}$ form a complete set it is necessary to check the Parseval identity

$$
\sum_{r, \pm}\left[\psi_{r}^{ \pm}(n)\right]^{*} \otimes \psi_{r}^{ \pm}\left(n^{\prime}\right)=\delta_{n, n^{\prime}} \hat{I}
$$

As it follows from Eq. (16),

$$
\psi_{r}^{ \pm}(n)=\psi_{n}^{ \pm}(r), \quad \Psi_{-r}^{+}(r)=\sigma_{y}\left[\psi_{r}^{-}(-n)\right]^{*} .
$$

Therefore, one arrives at the set of equalities

$$
\begin{aligned}
\sum_{r}\left[{\boldsymbol{\psi _ { r } ^ { - }}}^{-}(n)\right]^{*} & \otimes \boldsymbol{\psi}_{r}^{-}\left(n^{\prime}\right)=\sigma_{y} \sum_{r} \boldsymbol{\psi}_{r}^{+}(-n) \otimes\left[\boldsymbol{\psi}_{r}^{+}\left(-n^{\prime}\right)\right]^{*} \sigma_{y}= \\
& =\sigma_{y} \sum_{r} \boldsymbol{\psi}_{r}^{+}\left(n^{\prime}\right) \otimes\left[\boldsymbol{\psi}_{r}^{+}(n)\right]^{*} \sigma_{y}= \\
& =\sigma_{y}\left\{\sum_{n}\left[\boldsymbol{\psi}_{r}^{+}(n)\right]^{*} \otimes \boldsymbol{\psi}_{r}^{+}\left(n^{\prime}\right)\right\}^{T} \sigma_{y} .
\end{aligned}
$$

Having in mind, that

$$
\hat{X}+\sigma_{y} \hat{X}^{T} \sigma_{y}=\hat{I} \operatorname{Tr} \hat{X},
$$

one finally obtains

$$
\begin{gathered}
\sum_{r, \pm}\left[\boldsymbol{\psi}_{r}^{ \pm}(n)\right]^{*} \otimes \boldsymbol{\psi}_{r}^{ \pm}\left(n^{\prime}\right)=\hat{I} \sum_{r} \operatorname{Tr}\left[\boldsymbol{\psi}_{r}^{+}(n)\right]^{*} \otimes \boldsymbol{\psi}_{r}^{+}\left(n^{\prime}\right)= \\
=\hat{I} \sum_{r}\left(\boldsymbol{\Psi}_{n}^{+}(r), \boldsymbol{\psi}_{n^{\prime}}^{+}(r)\right)=\delta_{n, n^{\prime}} \hat{I}
\end{gathered}
$$

In this way the Parseval identity (A.1) is proved.

\section{Appendix B}

We search for a solution of (23) in the form

$$
B_{\beta}(n)=\mathrm{e}^{-i \pi a n^{2} / 2} \int_{0}^{2 \pi} d k \mathrm{e}^{-i k n} u_{\beta}(k) .
$$

Substituting (B.1) into (23) one gets the following equation for the $2 \pi$-periodic function $u_{\beta}(k)$ :

$$
u_{\beta}(k+\pi a)=\mathrm{e}^{i \lambda+i G(k+\lambda / 2)} u_{\beta}(k-\pi a),
$$

where

$$
G(k)=-i \ln \frac{1-i \tau \mathrm{e}^{-i k}}{1+i \tau \mathrm{e}^{i k}}, \quad \lambda \equiv \beta-\pi a
$$

The ways to solve (B.2) are different for irrational or rational $a$.

\section{a) Irrational a}

In this case one can look for the solution of (B.2) in the form

$$
u_{\beta}(k)=\exp \left[i k r+i \chi_{\beta}(k)\right]
$$

where $\chi_{\beta}(k)$ is $2 \pi$-periodic continuous function and $r$ is the arbitrary while number. $\chi_{\beta}(k)$ is determined by the following difference equation with constant coefficients:

$$
\chi_{\beta}(k+\pi a)-\chi_{\beta}(k-\pi a)=\lambda-2 \pi a r+G(k+\lambda / 2) .
$$


This equation has solutions if and only if the right part is orthogonal to all the solutions of uniform equation

$$
f(k+\pi a)-f(k-\pi a)=0 .
$$

If $a$ is an irrational number one can easily check that there is only one solution of Eq. (B.5) within the class of integrable functions, namely $f(k)=$ const. Keeping this fact in mind we integrate both parts of (B.4) over the variable $k$ from $-\pi$ to $\pi$ to find the value of $\lambda$ at which the solution of (B.4) exists. Substituting (B.3) for $G(k)$ we get $\lambda=\lambda_{r}=2 \pi a r$ and, as a consequence, the expression (24) for the eigenvalues of operator $\hat{T}$.

Solving Eq. (B.4) at $\lambda=\lambda_{r}$ by the Fourier method, one gets the following expression for the function $\chi(k)=\chi_{r}(k)$

$$
\chi_{r}(k)=-\sum_{l=1}^{\infty} \frac{\tau^{l}}{l} \frac{\cos l(k-\pi a r-\pi / 2)}{\sin \pi a l}
$$

It is known (see, e.g., Ref. 19) that for almost all irrational numbers $a$ (that means excluding a set of irrational numbers of zero measure) the inequality $|a-p / q|>C / q^{2}$ can be satisfied. Here $p, q$ are integer numbers, while $C$ depends only on $a$. One can show that this inequality leads to a convergence of the series (B.6) for almost all $a$. As a result, the function $u_{\beta}(k)$ can be analytically continued into the region

$$
\ln \tau<\operatorname{Im} k<-\ln \tau .
$$

Because of that the function $B_{\beta}(n)$ given by (B.1) decays as a $\tau^{-|n|}$ at $n \rightarrow \pm \infty$, and therefore the eigenstates of operator $\hat{T}$ are exponentially localized.

\section{b) Rational $a=p / q$}

In this case Eq. (23) is symmetric with respect to translation $n \rightarrow n+2 q$. Therefore its solution has the Bloch form, and as a consequence the function $u_{\beta}(k)$ defined by (B.1) appears singular. Thus at rational $a=p / q$ one can look for the solution of (B.2) in the form

$$
\begin{gathered}
u_{\beta}(k)=\exp [i k r+i \zeta(k)] \times \\
\times \sum_{m=0}^{q-1} \delta\left(\sin \left[\frac{k}{2}+\frac{\mathcal{K}}{4}-\pi a m+\frac{\pi a}{2}\right]\right),
\end{gathered}
$$

$\delta(x)$ is a delta function. Substituting this anzatz into the (B.2) and using the relationship

$$
\sum_{m=0}^{q-1} \delta\left[\sin \left(\frac{x}{2}-\pi a m\right)\right]=\sum_{m=0}^{q-1} \delta\left[\sin \frac{x}{2}+\pi a-\pi a m\right]
$$

we reconstruct it as

$$
\begin{gathered}
\mathcal{D}\{\zeta\} \sum_{m=0}^{q-1} \delta\left(\sin \left[\frac{k}{2}+\frac{\mathcal{K}}{4}-\pi a m\right]\right)=0 \\
\mathcal{D}\{\zeta\} \equiv \exp i \zeta(k+\pi a)-
\end{gathered}
$$

$-\exp i\left[\zeta(k-\pi a)+\lambda-2 \pi a r+G_{0}\left(k+\frac{\lambda}{2}\right)+\Lambda\left(\frac{\lambda-\mathcal{Z}}{2}\right)\right]$,

where

$$
\begin{gathered}
G(k)=G_{0}(k)+\Lambda(k), \\
\Lambda(k)=\frac{1}{2 \pi} \sum_{m} \int_{0}^{2 \pi} d k^{\prime} \mathrm{e}^{-i m q\left(k-k^{\prime}\right)} G\left(k^{\prime}\right)= \\
=\frac{2}{q} \arctan \frac{\tau^{q} \sin q(k-\pi / 2)}{1-\tau^{q} \cos q(k-\pi / 2)} .
\end{gathered}
$$

To obtain (B.8) we used the fact, that

$$
\begin{gathered}
\left\{\exp i \Lambda\left(k+\frac{\lambda}{2}\right)-\exp i \Lambda\left(\frac{\lambda-\mathcal{K}}{2}\right)\right\} \times \\
\quad \times \sum_{m=0}^{q-1} \delta\left[\sin \left(\frac{k}{2}+\frac{\mathcal{K}}{4}-\pi a m\right)\right]=0 .
\end{gathered}
$$

From Eq. (B.8) one can see that it is enough to find $\zeta(k)$ to satisfy the equation $\mathcal{D}\{\zeta\}=0$ and therefore, similarly to the case of irrational $a$, the problem is reduced to a difference equation with constant coefficients,

$$
\begin{gathered}
\zeta(k+\pi a)-\zeta(k-\pi a)= \\
=\lambda-2 \pi a r+G_{0}\left(k+\frac{\lambda}{2}\right)+\Lambda\left(\frac{\lambda-\mathcal{K}}{2}\right) .
\end{gathered}
$$

At rational $a=p / q$ Eq. (B.5) has the solution $f(k)=\exp i l q k, l=0, \pm 1, \pm 2, \ldots$ Consequently, the r.h.s. part of Eq. (B.9) must be orthogonal to this function rather than to a constant as in the case of irrational $a$. One can check that the integral of the r.h.s. part of (B.9) times $f(k)=\exp ($ ilqk $), l \neq 0$ vanishes for any $\lambda$. Integrating Eq. (B.9) over $k$ one gets the dispersion equation for $\lambda(\mathcal{K})$, 


$$
\lambda-2 \pi a r+\Lambda\left(\frac{\lambda-\mathcal{K}}{2}\right)=0 .
$$

If $\lambda(\mathcal{K})$ satisfies this equation, the solution of (B.9) is

$$
\zeta_{r}(k)=-\sum_{l=1}^{\infty} \tau^{l} \frac{\cos l(k-\pi a r-\pi / 2)}{\sin \pi a l}
$$

Here the prime indicates that the terms with $l=m q, m=1,2, \ldots$ are omitted.

From (B.10) one gets the following expression for $\lambda=\lambda_{r}(\mathcal{K})$ :

$\lambda_{r}(\mathcal{K})=2 \pi a r+\frac{2}{q} \arcsin \left[\tau^{q} \sin \frac{q}{2}(\mathcal{K}-2 \pi a r+\pi)\right]$,

for $r=0,1, \ldots, q-1$ and, as a consequence, the expression (27) for $\beta_{r}(\mathcal{K})$.

The expression for the Bloch function $\psi(n)$ corresponding to the eigenvalue $\lambda_{r=0}(\mathcal{K}) \equiv \lambda(\mathcal{K})$, has the form

$$
\begin{gathered}
\psi(n)=\mathrm{e}^{i \mathscr{K} n \mp i \pi a n(2 n \pm 1)} \times \\
\times \sum_{m=0}^{q-1} \mathrm{e}^{-4 i \pi a m n+i \xi_{m}^{-}}\left(\begin{array}{l}
f^{+}(\mathcal{K}) \mathrm{e}^{-i \xi_{m}^{-}+i \zeta\left(\xi_{m}^{+}\right)} \\
f^{-}(\mathcal{K}) \mathrm{e}^{-i \zeta\left(\xi_{m}^{-}-\pi a\right)}
\end{array}\right),
\end{gathered}
$$

where

$$
\begin{gathered}
\xi_{m}^{ \pm}(\mathcal{K})=-\frac{\mathcal{K}}{2}+2 \pi a m \pm \frac{\lambda(\mathcal{K})}{2} \\
f^{ \pm}(\mathcal{K})=\left[1 \pm \tau^{q} \frac{\cos q(\mathcal{K}+\pi) / 2}{\cos q \lambda(\mathcal{K}) / 2}\right]^{1 / 2}
\end{gathered}
$$

and $\zeta(k) \equiv \zeta_{r=0}(k)$ is defined by Eq. (B.11). In the limit $p, q \rightarrow \infty p / q=$ const one gets the expression (25) for the case of irrational $a$.

Thus, the expressions (B.11), (B.12), (B.13) together with (22) provide $2 q$ eigenstates of the operator $\hat{T}$ for a given value of $\mathcal{K}$ which plays the role of quasimomentum. Consequently, one obtains the complete set of eigenfunctions of operator $\hat{T}$ while $\mathcal{K}$ scans the interval $0 \leq \mathcal{K}<2 \pi / q$.

\section{Appendix C: Limit of strong localization}

To provide the strong localization limit $(\tilde{v} \rightarrow 0)$, one formulates the following statements.

Lemma 1: For unitary operator $\hat{P}$, defined according to

$$
\hat{P}=\hat{S} \sigma_{+} \otimes \mathrm{e}^{2 i \pi a \hat{n}}+\hat{S} \sigma_{-} \otimes \mathrm{e}^{i \pi \hat{a}} \hat{R_{-}} \mathrm{e}^{i \pi a \hat{n}},
$$

there is the following operator equality

$$
\hat{P^{+}} \hat{T} \hat{P}=\mathrm{e}^{2 i \pi a} \hat{T} .
$$

Consequently, operator $\hat{P}$ translates one of the eigenstates of the operator $\hat{T}(|\beta\rangle)$ to another state, $|\beta+2 \pi a\rangle$.

Lemma 2: The operators $\hat{T}, \hat{n}, \hat{P}, \hat{V}$ defined above, meet the operator equality

$$
\hat{V}=\hat{P}^{+} \hat{n} \hat{P}-\hat{T} \hat{P^{+}} \hat{n} \hat{P} \hat{T}^{+}
$$

(the proof can be obtained by direct substitution).

Basing on these statements one can express the matrix element $\Omega_{\beta}$ in Eq. (18) as

$$
\begin{aligned}
\Omega_{\beta} & =\left\langle\beta_{0}, \hat{V} \beta\right\rangle=\left[1-\mathrm{e}^{i\left(\beta_{0}-\beta\right)}\right]\left\langle\beta_{0}, \hat{P}^{+} \hat{n} \hat{P} \beta\right\rangle= \\
& =\left[1-\mathrm{e}^{i\left(\beta_{0}-\beta\right)}\right]\left\langle\left(\beta_{0}+2 \pi a\right), \hat{n}(\beta+2 \pi a)\right\rangle .
\end{aligned}
$$

Consequently, we can rewrite the Eq. (18) for the average current in the form

$$
\frac{I}{I_{0}}=4 \sum_{\beta}\left|\tilde{\Omega}_{\beta}\right|^{2} \frac{\tilde{v} \sin ^{2} \Phi_{\beta}}{\tilde{v}^{2}+\sin ^{2} \Phi_{\beta}},
$$

where

$$
\tilde{\Omega}_{\beta}=\sum_{n}\left(\Psi_{\beta_{0}}(n), \hat{n} \Psi_{\beta}(n)\right)
$$

Therefore, in the strong localization limit one has

$$
\begin{gathered}
\frac{I}{I_{0}}=4 \tilde{v} \sum_{n, \beta \neq \beta_{0}}\left(\Psi_{\beta_{0}}(n), \hat{n} \Psi_{\beta}(n)\right)\left(\Psi_{\beta}(n), \hat{n} \Psi_{\beta_{0}}(n)\right)= \\
=\tilde{v}\left(\frac{2}{\Delta} R_{\text {loc }}\right)^{2} .
\end{gathered}
$$

1. Y. Imry, Quantum Coherence in Mesoscopic Systems, Vol. 254 of NATO Advanced Study Institute, Series B: Physics, Plenum, New York (1991).

2. P. W. Anderson, Phys. Rev. 109, 1492 (1958).

3. B. L. Al'tshuler, Pis'ma Zh. Eksp. Teor. Fiz. 41, 530 (1985) [JETP Lett. 41, 648 (1985)].

4. M. Büttiker, Y. Imry, and R. Landauer, Phys. Lett. A96, 365 (1983)

5. R. Landauer, Phys. Rev. B33, 6497 (1983)

6. Y. Gefen and D. Thouless, Phys. Rev. Lett. 59, 1752 (1987)

7. Y. Gefen and D. J. Thouless, Philos. Mag. 56, 1005 (1987).

8. T. Swahn, E. N. Bogachek, Yu. M. Galperin, M. Jonson, and R. I. Shekhter, Phys. Rev. Lett. 73, 162 (1994).

9. E. Shimshoni and Y. Gefen, Annals of Physics 210, 16 (1991). 
10. R. Huibner and R. Graham, Phys. Rev. B53, 4870 (1996).

11. G. Casati, B. V. Chirikov, F. M. Izrailev, and J. Ford, in: Stochastic Behaviour in Classical and Quantum Hamiltonian Systems, G. Casati and J. Ford (eds.), Lecture in Physics, Vol. 93, Springer, Berlin (1979), p. 334.

12. D. R. Grempel, S. Fishman, and R. E. Prange, Phys. Rev. A29, 1639 (1984).

13. G. Blatter and D. A. Browne, Phys. Rev. B37, 3856 (1988).

14. P. Ao, Phys. Rev. B41, 3998 (1990).

15. A brief account of these ideas has been published: L. Y. Gorelik, S. Kulinich, Yu. Galperin, R. I. Shekhter, and M. Jonson, Phys. Rev. Lett. 78, 2196 (1997).
16. L. D. Landau, Phys. Z. Sov. 2, 46 (1932); C. Zener, Proc. Roy. Soc. (London) A137, 696 (1932); K. Mullen, E. Ben-Jacob, Y. Gefen, and Z. Schuss, Phys. Rev. Lett. 62, 2543 (1989).

17. When integrating over time in (11) we exclude small time intervals of order $\sqrt{t_{0} \hbar / \Delta}$ near the points $-t_{0} / 2,0, t_{0} / 2$, where the commutator $\left[\mathcal{H}(t), \mathcal{H}\left(t^{\prime}\right)\right]$ does not vanish.

18. This expression was derived for $q=1$ in Ref. 13 .

19. See for example J. W. S. Cassels, An Introduction to Diophantine Approximation, Cambridge University Press (1957). 